\title{
Assessment of cohesive traction-separation relationships in ABAQUS: A comparative study
}

\author{
Kyoungsoo Park ${ }^{1 *}$; Habeun Choi ${ }^{1}$; Glaucio H. Paulino ${ }^{2}$
}

\begin{abstract}
The definition of a traction-separation relationship is essential in cohesive zone models because it describes the nonlinear fracture process zone. A few models are investigated in this paper and a comparative study is conducted. Among various traction-separation relationships, the one in Abaqus is assessed by evaluating the cohesive traction and its tangent stiffness according to a given separation path. The results demonstrate that the traction-separation relationship in Abaqus can lead to non-physical responses because of a pathological positive tangent stiffness under softening condition. This is reflected in cohesive tractions that increase and decrease repeatedly while the cohesive separation monotonically increases. Thus, together with supporting information, this paper conveys the message that a traction-separation relationship should be developed and selected with great caution, especially under mixed-mode conditions.
\end{abstract}

Keywords: Abaqus, Cohesive zone model, PPR model, Traction-separation relationship, Nonlinear fracture mechanics

\section{Introduction}

Cohesive zone models have been extensively utilized to represent various nonlinear material fracture behaviors. In many instances, cracks propagate under mixed-mode conditions rather than, for example, isolated mode-I or mode-II. In fact, different choices of the traction-separation relationships can result in different failure behavior and structural response (Campilho et al., 2013; Chandra et al., 2002). Thus, consistent representation of traction-separation relationships under mixed-mode conditions is critical for proper use and interpretation of cohesive zone models.

In general, traction-separation relationships can be classified into potential-based models and nonpotential-based models. Potential-based models use the concept of cohesive energy potential - see, for example, Needleman (1987). The gradient of the cohesive energy potential leads to the cohesive traction versus separation relationships within the softening region. Several potential expressions have been proposed in conjunction with fracture parameters such as fracture energy and cohesive strength (e.g. Needleman, 1990; Tvergaard and Hutchinson, 1992; Xu and Needleman,

*Corresponding author: Email: k-park@yonsei.ac.kr, Phone: +82-2-2123-5806
1993). Following the framework of potential-based models, the so-called PPR (Park-Paulino-Roesler) model was established on the basis of consistent cohesive fracture boundary conditions under mixedmode conditions (Park et al., 2009). Since its publication, this model has been investigated in several physical contexts including multiscale fracture (Cerrone et al. 2014), fragmentation (Paulino et al., 2010; Leon et al., 2014; Spring et al., 2014), and composite material failure (Neto et al. 2016). The PPR model has also been independently implemented in Abaqus as user-defined elements (Park and Paulino, 2012; Spring and Paulino, 2014). For an in-depth review of potential-based models, the reader is referred to Park and Paulino (2013).

For non-potential-based models, tractionseparation models with various shapes have been developed, e.g. linear softening (Camanho et al., 2003), trapezoidal shape (Yang and Thouless, 2001), bilinear softening (Wittmann et al., 1988), trilinear softening (Park et al., 2010), and exponential models (McGarry et al., 2014) to name a few. These models do not need to satisfy the symmetry condition, i.e., an exact differential with $\partial T_{n} / \Delta_{t}=\partial T_{t} / \Delta_{n}$. However, nonpotential-based models may not provide a consistent traction-separation relationship, and they do not account for all possible separation paths within the softening region. 
The traction-separation model proposed by Camanho et al. (2003) has been extensively utilized to investigate mixed-mode fracture behavior (e.g. Lee et al., 2010; Morais and Moura, 2006; Sørensen et al., 2009; Yang et al., 2009). This model was implemented in the commercial software Abaqus (2013) and was reformulated on the basis of cohesive energy potential and damage mechanics (Turon et al., 2006). A similar traction-separation relationship was also developed for analyzing delamination of composite materials (Jiang et al., 2007; Harper and Stephen, 2008). Recently, McGarry et al. (2014) proposed three non-potentialbased models while addressing the relative weight of mode-mixity (Máirtín et al., 2014). However, such traction-separation relationships are prone to inconsistency, for instance, a positive stiffness under softening conditions.

In this context, the consistency of the nonpotential-based models, including the aforementioned Abaqus model, is assessed, while several limitations of the models are addressed through comparison with the PPR reference model (Park et al., 2009). The remainder of the paper is organized as follows. Section 2 briefly describes five traction-separation relations, i.e. the Abaqus model, the three models by McGarry et al. (2014), and the PPR model. In Section 3, the consistency of the models is verified by evaluating the tangent stiffness of the traction-separation relation. Finally, some key findings of the paper are summarized in Section 4.

\section{Cohesive Zone Model}

In the cohesive zone model, the nonlinear fracture process zone is approximated by means of cohesive surface elements that follow a traction-separation relation, which are inserted along fracture surfaces. In the commercial software Abaqus, the cohesive elements are inserted a priori between bulk elements (often along a predefined path), leading to the so-called intrinsic cohesive zone model. The intrinsic tractionseparation relationships of Abaqus, McGarry et al. and PPR models are briefly explained below.

\subsection{Traction-Separation Relationship in ABAQUS}

The traction-separation relationship in Abaqus was originally developed by Camanho et al. (2003). This model considers the variation of fracture toughness as a function of a mode-mixity ratio. In order to account for the mixed-mode condition, an effective separation $(\bar{\Delta})$ is defined as

$$
\bar{\Delta}=\sqrt{\Delta_{n}^{2}+\Delta_{t}^{2}}
$$

where $\Delta_{n}$ and $\Delta_{t}$ are the normal and tangential separations, respectively. When the effective separation is smaller than an effective critical separation $\left(\bar{\delta}_{c}\right)$, the normal and tangential tractions $\left(T_{n}, T_{t}\right)$ are proportional to the normal and tangential separations, which are given as

$$
T_{n}=K_{p} \Delta_{n}, T_{t}=K_{p} \Delta_{t}
$$

where $K_{p}$ is a penalty stiffness. When the separations reach the effective critical separation, the state of separation corresponds to the onset of damage and/or crack initiation. A quadratic failure criterion is employed to determine the onset of damage, which leads to the following effective critical separation, i.e.

$$
\bar{\delta}_{c}=\delta_{n c} \delta_{t c} \sqrt{\frac{1+\beta_{e}{ }^{2}}{\delta_{t c}{ }^{2}+\left(\beta_{e} \delta_{n c}\right)^{2}}}
$$

where $\delta_{n c}$ and $\delta_{t c}$ are defined as the ratio between the normal and tangential cohesive strengths $\left(\sigma_{\max }\right.$, $\left.\tau_{\max }\right)$ and the penalty stiffness, respectively, and $\beta_{e}$ is the ratio between the tangential and normal separations. Note that when the normal cohesive strength is the same as the tangential cohesive strength, the critical separation is a constant, i.e. $\bar{\delta}_{c}=\sigma_{\max } / K_{p}$, because the $\beta_{e}$ term cancels out.

When the effective separation is greater than the effective critical separation $\left(\bar{\delta}_{c}\right)$ and smaller than the effective complete failure separation $\left(\bar{\delta}_{f}\right)$, i.e. $\bar{\delta}_{c}<\bar{\Delta}<\bar{\delta}_{f}$, the state of separation corresponds to the softening condition. Then, the normal and tangential cohesive tractions are defined as

$$
\begin{aligned}
T_{n} & =(1-d) K_{p} \Delta_{n} \\
T_{t} & =(1-d) K_{p} \Delta_{t}
\end{aligned}
$$

where $d$ is a scalar damage variable. Two types of damage evolution are available in Abaqus: linear and exponential models. Accordingly, the expressions for the linear and exponential damage models are given as

and

$$
d=\frac{\bar{\delta}_{f}\left(\bar{\Delta}-\bar{\delta}_{c}\right)}{\bar{\Delta}\left(\bar{\delta}_{f}-\bar{\delta}_{c}\right)}
$$

$$
d=1-\left(\frac{\bar{\delta}_{c}}{\bar{\Delta}}\right)\left[1-\frac{1-\exp \left(-\alpha_{r}\left(\frac{\bar{\Delta}-\bar{\delta}_{c}}{\bar{\delta}_{f}-\bar{\delta}_{c}}\right)\right)}{1-\exp \left(-\alpha_{r}\right)}\right]
$$

respectively, where $\alpha_{r}$ is a parameter associated with the rate of damage evolution. The damage variable is initially zero, and monotonically increases to the numeric value of one under softening condition. Additionally, the effective complete failure separation is evaluated by employing the mixed-mode failure 
criterion proposed by Benzeggagh and Kenane (1996), which leads to the following expression:

$$
\bar{\delta}_{f}=\frac{2}{K_{p} \bar{\delta}_{c}}\left[\phi_{n}+\left(\phi_{t}-\phi_{n}\right)\left(\frac{\beta_{e}{ }^{2}}{1+\beta_{e}{ }^{2}}\right)^{\eta}\right]
$$

where $\eta$ is a material parameter associated with the variation of fracture toughness. When the normal and tangential fracture energies are the same, the effect of $\eta$ disappears, and the complete failure separation reduces to $\bar{\delta}_{f}=2 \phi_{n} /\left(K_{p} \bar{\delta}_{c}\right)$. If the effective separation is greater than the effective complete failure separation, then both normal and tangential cohesive tractions are zero, as expected. Notice that when the normal and tangential fracture energies and cohesive strengths are the same, the traction-separation relationship in Abaqus simplifies to the model proposed by Tvergaard (1990).

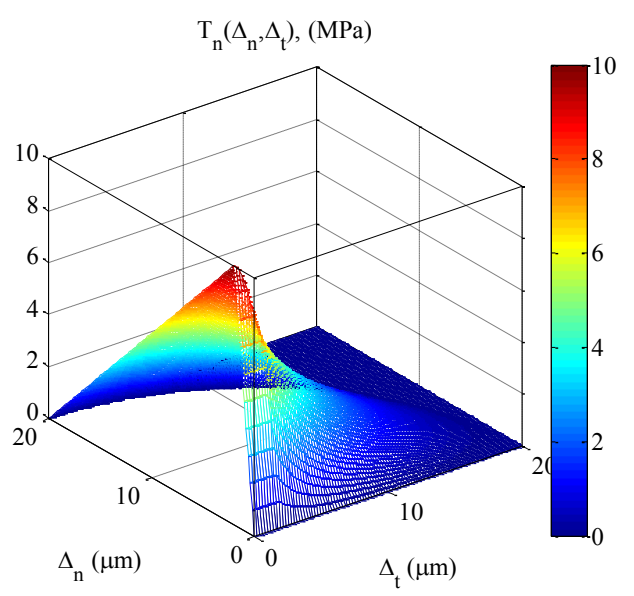

(a)

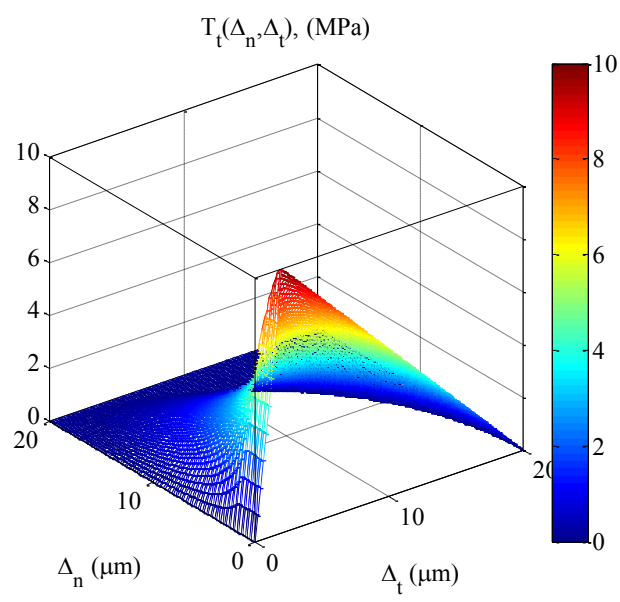

(b)

Fig. 1. Illustration of the Abaqus CZM: (a) Normal and (b) tangential cohesive tractions with $\phi_{n}=100 \mathrm{~J} / \mathrm{m}^{2}$, $\phi_{t}=100 \mathrm{~J} / \mathrm{m}^{2}, \sigma_{\max }=10 \mathrm{MPa}, \tau_{\max }=10 \mathrm{MPa}$, and $K_{p}=5 \mathrm{MPa} / \mu \mathrm{m}$.
To illustrate the aforementioned description, the normal and tangential tractions of the Abaqus model are plotted in Figure 1. The linear damage model is used, and thus the normal and tangential cohesive tractions linearly decrease along the mode-I and modeII directions. For the parametric study to be conducted, Mode-I fracture parameters are arbitrarily selected as the fracture energy of $100 \mathrm{~J} / \mathrm{m}^{2}$ and the cohesive strength of $10 \mathrm{MPa}$. The mode-II fracture parameters are assumed to be the same as the mode-I parameters, leading to constant values of the effective critical separation and the complete failure separation with respect to the mode-mixity. In addition, the penalty stiffness is selected as $5 \mathrm{MPa} / \mu \mathrm{m}$, which results in $\bar{\delta}_{c} / \bar{\delta}_{f}=0.1$ where $\bar{\delta}_{c}$ and $\bar{\delta}_{f}$ are $2 \mu \mathrm{m}$ and $20 \mu \mathrm{m}$, respectively, in this example.

\subsection{Non-potential-based models by McGarry et al. (2014)}

Based on the exponential potential by $\mathrm{Xu}$ and Needleman (1993), McGarry et al. (2014) proposed three non-potential-based models. The non-potentialbased formulation 1 (NP1) is expressed as

$$
\begin{aligned}
& T_{n}\left(\Delta_{n}, \Delta_{t}\right) \\
& =\sigma_{\text {max }} \exp (1)\left(\frac{\Delta_{n}}{\delta_{n}}\right) \exp \left(-\frac{\Delta_{n}}{\delta_{n}}\right) \exp \left(-\frac{\Delta_{t}^{2}}{\delta_{t}^{2}}\right)
\end{aligned}
$$

and

$$
\begin{aligned}
& T_{t}\left(\Delta_{n}, \Delta_{t}\right) \\
& =\tau_{\max } \sqrt{2 \exp (1)}\left(\frac{\Delta_{t}}{\delta_{t}}\right) \exp \left(-\frac{\Delta_{n}}{\delta_{n}}\right) \exp \left(-\frac{\Delta_{t}^{2}}{\delta_{t}^{2}}\right)
\end{aligned}
$$

where the characteristic length $\left(\delta_{n}, \delta_{t}\right)$ is evaluated from the fracture energy. In order to provide equivalent normal and tangential traction-separation relationships, the second non-potential-based formulation (NP2) is given as

$$
\begin{aligned}
& T_{n}\left(\Delta_{n}, \Delta_{t}\right) \\
& =\sigma_{\max } \exp (1)\left(\frac{\Delta_{n}}{\delta_{n}}\right) \exp \left(-\frac{\Delta_{n}}{\delta_{n}}\right) \exp \left(-\alpha_{m} \sqrt{\frac{\Delta_{t}^{2}}{\delta_{t}^{2}}}\right)
\end{aligned}
$$

and

$$
\begin{aligned}
& T_{t}\left(\Delta_{n}, \Delta_{t}\right) \\
& =\tau_{\text {max }} \exp (1)\left(\frac{\Delta_{t}}{\delta_{t}}\right) \exp \left(-\sqrt{\frac{\Delta_{t}^{2}}{\delta_{t}^{2}}}\right) \exp \left(-\beta_{m} \frac{\Delta_{n}}{\delta_{n}}\right)
\end{aligned}
$$

where $\alpha_{m}$ and $\beta_{m}$ are associated with the weight of the mode-mixity. Finally, based on the effective separation, the third non-potential-based formulation (NP3), also named as separation magnitude coupling (SMC) formulation, is given as

$$
\begin{aligned}
& T_{n}\left(\Delta_{n}, \Delta_{t}\right) \\
& =\sigma_{\max } \exp (1)\left(\frac{\Delta_{n}}{\delta_{n}}\right) \exp \left(-\sqrt{\frac{\Delta_{n}^{2}}{\delta_{n}^{2}}+\frac{\Delta_{t}^{2}}{\delta_{t}^{2}}}\right)
\end{aligned}
$$


and

$$
\begin{aligned}
& T_{t}\left(\Delta_{n}, \Delta_{t}\right) \\
& =\tau_{\max } \exp (1)\left(\frac{\Delta_{t}}{\delta_{t}}\right) \exp \left(-\sqrt{\frac{\Delta_{n}^{2}}{\delta_{n}^{2}}+\frac{\Delta_{t}^{2}}{\delta_{t}^{2}}}\right)
\end{aligned}
$$

Note that for the case of $\delta_{n}=\delta_{t}$, the NP3 formulation is a special case of the model proposed by Tvergaard (1990). For further details of the models NP1, NP2 and NP3, the reader is referred to McGarry et al. (2014).

\subsection{PPR model}

In order to provide consistent cohesive tractionseparation relationships, the PPR model by Park et al. (2009) has been formulated on the basis of a fracture energy potential. The potential of the PPR model is given as

$$
\begin{aligned}
& \Psi\left(\Delta_{n}, \Delta_{t}\right)=\min \left(\phi_{n}, \phi_{t}\right) \\
& +\left[\Gamma_{n}\left(1-\frac{\Delta_{n}}{\delta_{n}}\right)^{\alpha}\left(\frac{m}{\alpha}+\frac{\Delta_{n}}{\delta_{n}}\right)^{m}+\left\langle\phi_{n}-\phi_{t}\right\rangle\right] \\
& \times\left[\Gamma_{t}\left(1-\frac{\left|\Delta_{t}\right|}{\delta_{t}}\right)^{\beta}\left(\frac{n}{\beta}+\frac{\left|\Delta_{t}\right|}{\delta_{t}}\right)^{n}+\left\langle\phi_{t}-\phi_{n}\right\rangle\right]
\end{aligned}
$$

where $\langle\cdot\rangle$ is the Macaulay bracket. Characteristic parameters $\left(\Gamma_{n}, \Gamma_{t} ; \delta_{n}, \delta_{t} ; m, n ; \alpha, \beta\right)$ are explicitly obtained from actual cohesive fracture parameters such as fracture energy, cohesive strength, shape of interactions, and initial ascending slope for each fracture mode. The expressions for the characteristic parameters are given in the original paper by Park et al. (2009). The gradient of the potential leads to the normal and tangential tractions, expressed as

$$
\begin{gathered}
T_{n}\left(\Delta_{n}, \Delta_{t}\right)=\frac{\Gamma_{n}}{\delta_{n}}\left[m\left(1-\frac{\Delta_{n}}{\delta_{n}}\right)^{\alpha}\left(\frac{m}{\alpha}+\frac{\Delta_{n}}{\delta_{n}}\right)^{m-1}\right. \\
\left.-\alpha\left(1-\frac{\Delta_{n}}{\delta_{n}}\right)^{\alpha-1}\left(\frac{m}{\alpha}+\frac{\Delta_{n}}{\delta_{n}}\right)^{m}\right] \\
\times\left[\Gamma_{t}\left(1-\frac{\left|\Delta_{t}\right|}{\delta_{t}}\right)^{\beta}\left(\frac{n}{\beta}+\frac{\left|\Delta_{t}\right|}{\delta_{t}}\right)^{n}+\left\langle\phi_{t}-\phi_{n}\right\rangle\right]
\end{gathered}
$$

and

$$
\begin{gathered}
T_{t}\left(\Delta_{n}, \Delta_{t}\right)=\frac{\Gamma_{t}}{\delta_{t}}\left[n\left(1-\frac{\left|\Delta_{t}\right|}{\delta_{t}}\right)^{\beta}\left(\frac{n}{\beta}+\frac{\left|\Delta_{t}\right|}{\delta_{t}}\right)^{n-1}\right. \\
\left.-\beta\left(1-\frac{\left|\Delta_{t}\right|}{\delta_{t}}\right)^{\beta-1}\left(\frac{n}{\beta}+\frac{\left|\Delta_{t}\right|}{\delta_{t}}\right)^{n}\right] \\
\times\left[\Gamma_{n}\left(1-\frac{\Delta_{n}}{\delta_{n}}\right)^{\alpha}\left(\frac{m}{\alpha}+\frac{\Delta_{n}}{\delta_{n}}\right)^{m}+\left\langle\phi_{n}-\phi_{t}\right\rangle\right] .
\end{gathered}
$$

In general, the cohesive tractions increase from zero to their maximum value when the cohesive separations go from zero to the critical separations $\left(\delta_{n c}, \delta_{t c}\right)$. Then, the tractions monotonically decrease to zero in a softening region when the cohesive separations reach complete failure separations $\left(\delta_{n}, \delta_{t}\right)$. Note that the cohesive tractions (Eqs. (15) and (16)) are defined within the cohesive interaction region, which is associated with the complete failure conditions. If the separation is outside of the cohesive interaction region, the cohesive tractions are zero. Additionally, the cohesive interaction region consists of two parts, i.e. elastic region and softening region. The boundary between the elastic region and the softening region is defined by the critical separation.

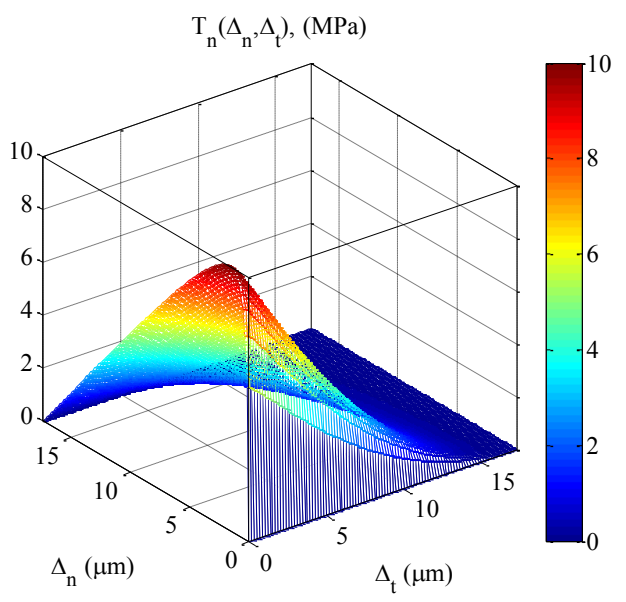

(a)

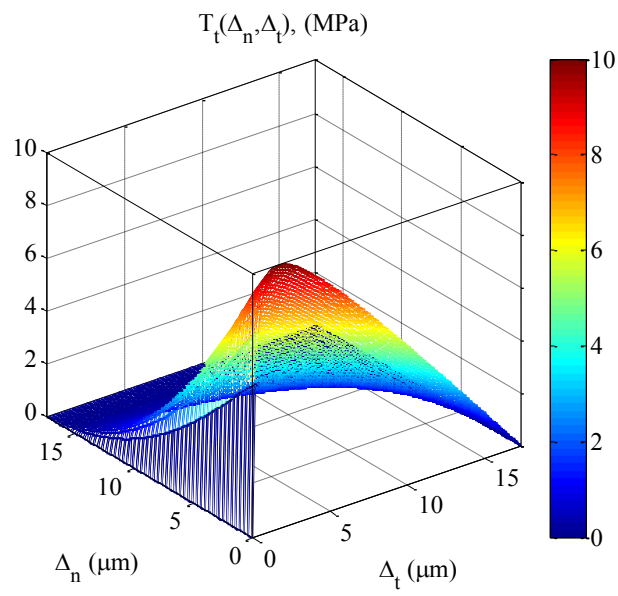

(b)

Fig. 2. PPR Model: (a) Normal and (b) tangential cohesive tractions with $\phi_{n}=100 \mathrm{~J} / \mathrm{m}^{2}, \phi_{t}=100$ $\mathrm{J} / \mathrm{m}^{2}, \sigma_{\text {max }}=10 \mathrm{MPa}, \tau_{\text {max }}=10 \mathrm{MPa}, \alpha=2, \beta=$ $2, \lambda_{n}=0.1$, and $\lambda_{t}=0.1$.

To illustrate, the normal and tangential tractions of the PPR model are plotted in Figure 2 within the cohesive interaction region. For mode-I, the fracture energy $\left(\phi_{n}\right)$ and the cohesive strength $\left(\sigma_{\max }\right)$ are 100 $\mathrm{J} / \mathrm{m}^{2}$ and $10 \mathrm{MPa}$, respectively. The initial slope 
indicator $\left(\lambda_{n}\right)$, i.e. the ratio of the critical separation $\left(\delta_{n c}\right)$ to the complete separation $\left(\delta_{n}\right)$, is selected as 0.1 . The shape parameter $(\alpha)$ is 2 , which provides nearly linear softening behavior. The fracture parameters of the tangential mode $\left(\phi_{t}, \tau_{\max }, \lambda_{t}, \beta\right)$ are assumed to be the same as the fracture parameters of mode-I.

\section{On Consistency of Traction-Separation Relationships}

One of the fundamental requirements for a consistent traction-separation relationship is that the cohesive traction decreases according to the increase of the separation across fracture surfaces, which results in a negative tangent stiffness under the softening condition (Elices et al., 2002; Park and Paulino, 2013). This is because the wider the separation is, the more the damage that occurs, and thus less resistance is expected along the fracture surfaces. In order to assess the consistency of the traction-separation relationships of Abaqus, NP1, NP2, NP3, and PPR models, the cohesive traction is evaluated for an arbitrary separation path. Then, whenever non-physical behavior occurs, it is explained by calculating the tangent stiffness of the traction-separation relations under the softening condition. Finally, the onset of softening is evaluated to demonstrate the consistency of the traction-separation relationships.

\subsection{Traction-separation relationship for an arbitrary separation path}

An arbitrary separation path, as shown in Figure 3, is selected to evaluate the aforementioned cohesive zone models (discussed in Section 2). The cohesive fracture parameters used to assess the cohesive zone models are as described in Figures 1 and 2. For the tractionseparation relationships, the fracture energy and the cohesive strength are $100 \mathrm{~J} / \mathrm{m}^{2}$ and $10 \mathrm{MPa}$, respectively, and the ratio of the critical separation to the complete failure separation is 0.1. Additionally, the critical separations of the Abaqus and PPR models are $2 \mu \mathrm{m}$ and $1.732 \mu \mathrm{m}$, respectively, while of the NP1, NP2 and NP3 models are not clearly defined.

For the separation path shown in Figure 3, both normal and tangential separations initially increase with a magnitude of $2 \mu \mathrm{m}$, which corresponds to Point $\mathrm{S}_{\mathrm{nt}}$. Next, the tangential separation increases an amount of $1 \mu \mathrm{m}$ (Point $\mathrm{S}_{\mathrm{t} 1}$ ), and then the normal separation increases by $1 \mu \mathrm{m}$ (Point $\mathrm{S}_{\mathrm{n} 1}$ ). Afterwards, this step is repeated until the state of separation reaches the complete failure condition. Because the specified separation monotonically increases, one expects a monotonic increase of damage, resulting on a monotonic decrease of the cohesive traction within the softening region. If a separation path is within the elastic region, i.e. the initial ascending part, the cohesive traction can increase while the separation increases, as expected.

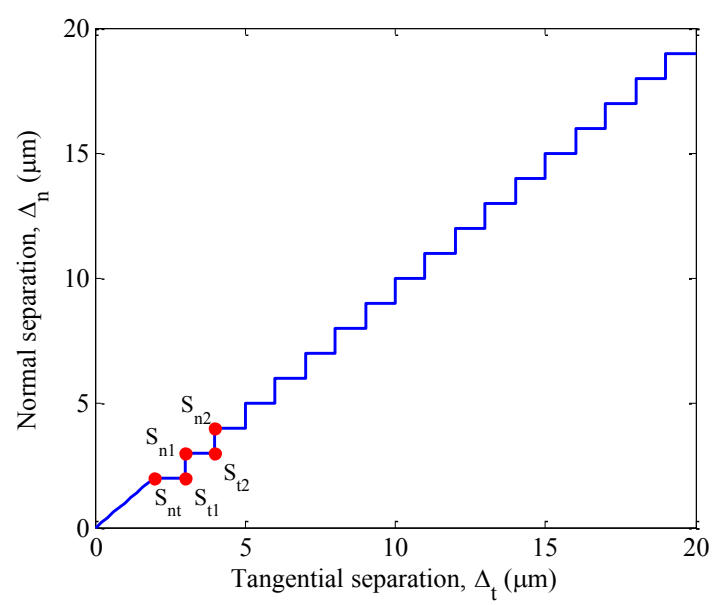

Fig. 3. Arbitrary separation path for evaluating normal and tangential cohesive tractions of various cohesive zone models.

Based on the specified separation path, the normal and tangential cohesive tractions are plotted in Figures 4 and 5 for both the Abaqus and the PPR models. The normal and tangential cohesive tractions initially increase up to the critical separation, and then monotonically decrease until normal and tangential separations reach Point $S_{n t}$ for both models. This is because the state of separation at Point $S_{n t}$ corresponds to the softening condition. Note that, for the Abaqus model, the effective separation $(\bar{\Delta})$ is $2.828 \mu \mathrm{m}$, which is greater than the effective critical separation (i.e. $\overline{\delta_{c}}$ $=2 \mu \mathrm{m})$. For the PPR model, the normal and tangential separations (i.e. $\Delta_{n}=\Delta_{t}=2 \mu \mathrm{m}$ ) are also greater than the critical normal and tangential separations (i.e. $\delta_{n c}=$ $\left.\delta_{t c}=1.732 \mu \mathrm{m}\right)$.

After reaching Point $S_{n}$, the cohesive traction response of the Abaqus model is different from the response of the PPR model. For the Abaqus model, when the tangential separation increases from $2 \mu \mathrm{m}$ to $3 \mu \mathrm{m}$ (from Point $S_{\mathrm{nt}}$ to Point $\mathrm{S}_{\mathrm{t} 1}$ ), the normal traction decreases (see Figure 4(b)) while the tangential traction increases (see Figure 5(b)). Note that the increase of the cohesive traction with respect to the increase of separation is not realistic because a larger separation should be associated with more damage and less resistance along the fracture surfaces. Next, when the normal separation increases from $2 \mu \mathrm{m}$ to $3 \mu \mathrm{m}$ (from 
Point $S_{t 1}$ to Point $S_{n 1}$ ), the normal traction increases (see Figure 4(a)) and the tangential traction decreases (see Figure 5(a)), in the Abaqus model. These cohesive traction fluctuations are non-physical, and persist until the normal and tangential separations approximately reach $6.75 \mu \mathrm{m}$ and $7.25 \mu \mathrm{m}$, respectively. When the normal and tangential separations are greater than these values, then both normal and tangential tractions decrease (in this example). However, for the PPR model, both normal and tangential separations monotonically decrease within the entire softening region.

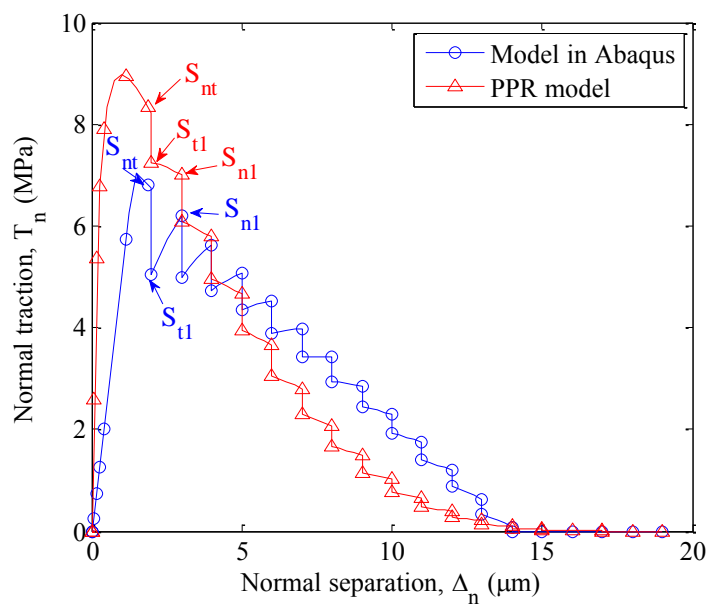

(a)

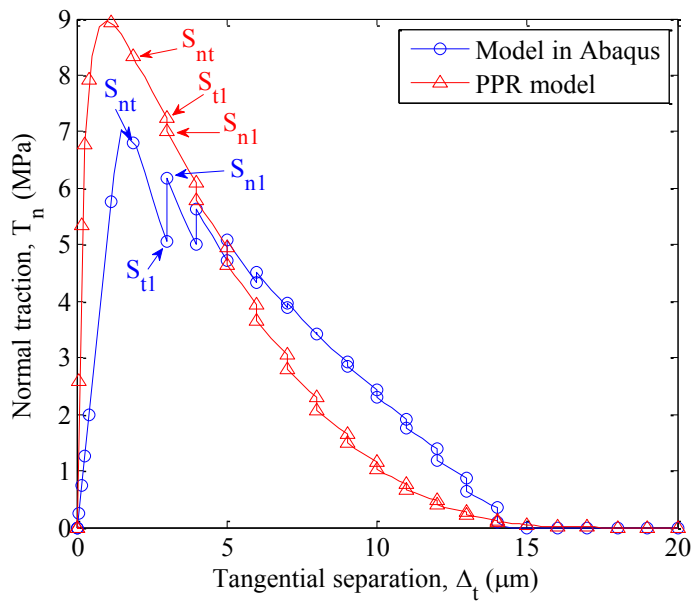

(b)

Fig. 4. Normal cohesive traction with respect to (a) normal separation and (b) tangential separation.

The oscillation of the cohesive tractions in the Abaqus model is associated with the competition between the damage variable and a linear separation term. The first tem in Eq. (4), i.e. $(1-d)$, contributes to the decrease of the cohesive traction because the damage variable $(d)$ monotonically increases according to the increase of the separation variable. The linear separation terms in Eq (4), i.e. $\Delta_{n}$ and $\Delta_{t}$, contribute to the increase of the cohesive traction. Then, when the rate of change of the linear separation term is greater than the rate of the change of the damage variable term, the cohesive traction increases with the increase of the cohesive separation. In order to confirm such behavior, the tangent stiffness under the softening region is evaluated in the following subsection.

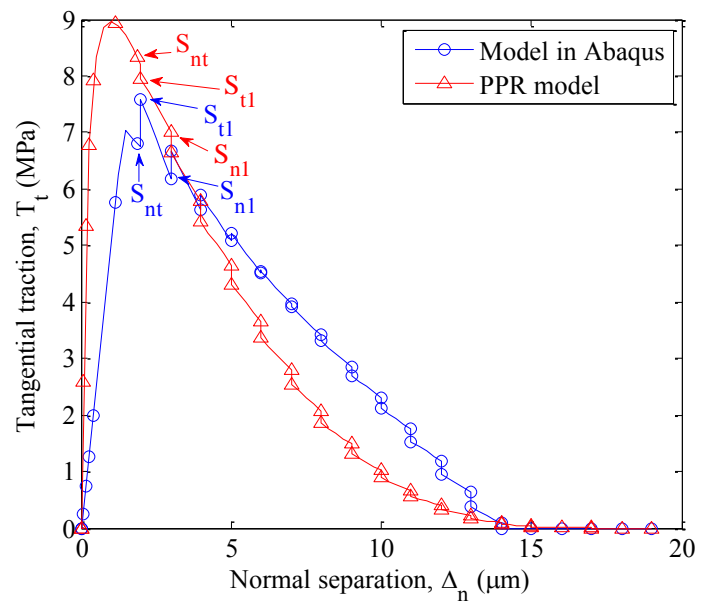

(a)

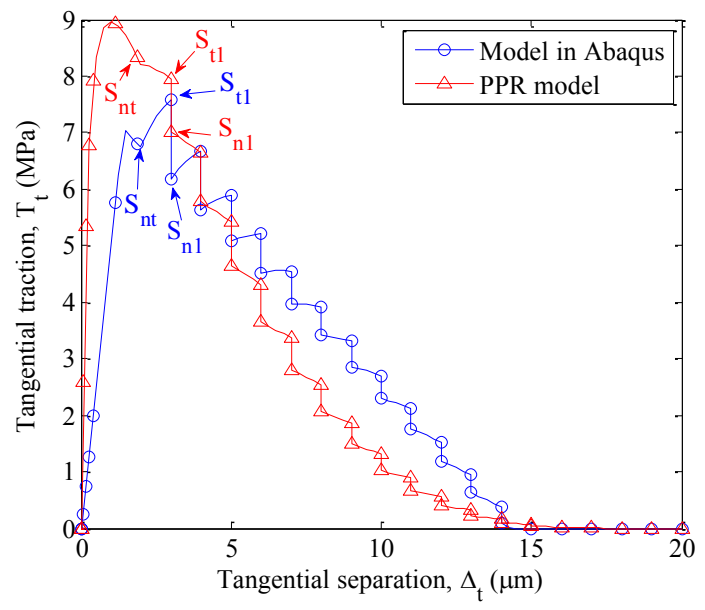

(b)

Fig. 5. Tangential cohesive traction with respect to (a) normal separation and (b) tangential separation. 
Additionally, the cohesive traction-separations of the non-potential-based models, i.e. NP1, NP2 and NP3, are evaluated for the same separation path, as shown in Figure 6. Similarly to the Abaqus model, the nonpotential-based models demonstrate the oscillation of the cohesive traction according to the increase of separation. Note that the oscillation is also associated with the competition between the linear separation term (i.e. $\Delta_{n}$ and $\Delta_{t}$ ) and the exponential decaying terms in the non-potential-based models.

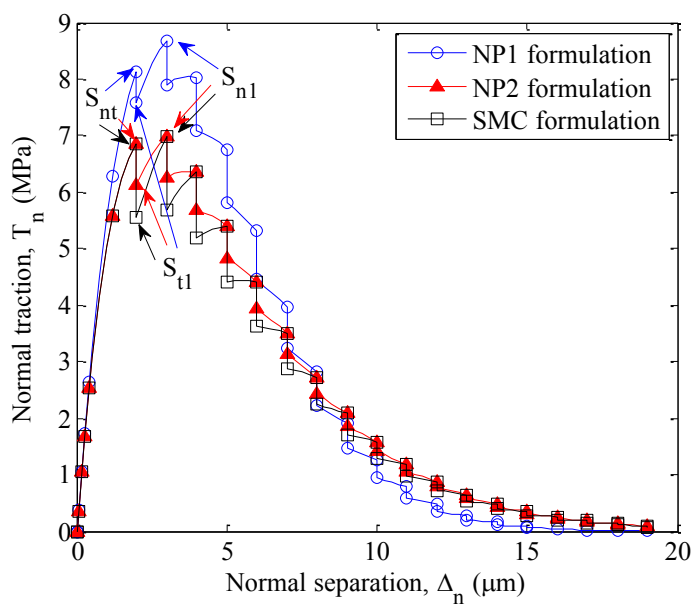

(a)

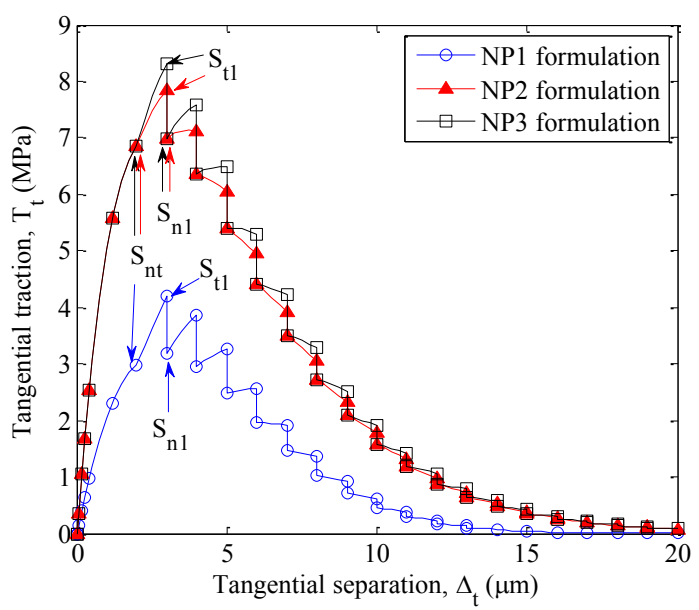

(b)

Fig. 6. Cohesive traction of the non-potential-based models: (a) normal traction and (b) tangential traction.

\subsection{Tangent stiffness under the softening region}

In order to confirm the non-physical behavior of the Abaqus model, the tangent stiffness is evaluated in the softening region. For this purpose, we employ the chain rule to obtain the derivative of the damage variable from Eq. (5) with respect to the normal separation, i.e.

$$
\frac{\partial d}{\partial \Delta_{n}}=\frac{\partial d}{\partial \bar{\Delta}} \frac{\partial \bar{\Delta}}{\partial \Delta_{n}}+\frac{\partial d}{\partial \bar{\delta}_{c}} \frac{\partial \overline{\delta_{c}}}{\partial \Delta_{n}}+\frac{\partial d}{\partial \bar{\delta}_{f}} \frac{\partial \overline{\delta_{f}}}{\partial \Delta_{n}}
$$

because $\bar{\Delta}, \overline{\delta_{c}}$ and $\overline{\delta_{f}}$ are expressed in terms of the normal and tangential separations. Alternatively, Turon et al. (2006) assumed that the variations of the critical separation and the complete failure separation were not significant, and thus only considered the first term in Eq. (17) for the derivative of the damage variable. Using Eq. (17), the derivative of the normal traction (Eq. (4)) with respect to the normal separation is given as

$$
\begin{aligned}
& \frac{\partial T_{n}}{\partial \Delta_{n}}=K_{p}(1-d)-\frac{K_{p} \Delta_{n}^{2} \overline{\delta_{c}} \overline{\delta_{f}}}{\bar{\Delta}^{3}\left(\overline{\delta_{f}}-\overline{\delta_{c}}\right)} \\
& \left(1+\frac{\Delta_{t}^{2}\left(\overline{\delta_{c}}+\overline{\delta_{f}}-2 \bar{\Delta}\right)}{\bar{\Delta}^{2}\left(\overline{\delta_{f}}-\overline{\delta_{c}}\right)}\left(\frac{\bar{\delta}_{c}^{2}}{\delta_{n c}^{2}}-\frac{\bar{\delta}_{c}^{2}}{\delta_{t c}^{2}}\right)\right) \\
& -\frac{2 \eta d \Delta_{n}^{2} \overline{\delta_{c}}}{\bar{\Delta}^{2}\left(\overline{\delta_{f}}-\overline{\delta_{c}}\right)}\left(K_{p}-\frac{2 \phi_{n}}{\overline{\delta_{c}} \bar{\delta}_{f}}\right)
\end{aligned}
$$

within the softening condition, i.e. $\bar{\delta}_{c}<\bar{\Delta}<\bar{\delta}_{f}$.

For the PPR model, the complete failure separation and the characteristic parameters are obtained from the fracture parameters, and thus the derivative of the normal traction with respect to the normal separation is expressed as

$$
\begin{aligned}
\frac{\partial T_{n}}{\partial \Delta_{n}}= & \frac{\Gamma_{n}}{\delta_{n}^{2}}\left[\left(m^{2}-m\right)\left(1-\frac{\Delta_{n}}{\delta_{n}}\right)^{\alpha}\left(\frac{m}{\alpha}+\frac{\Delta_{n}}{\delta_{n}}\right)^{m-2}\right. \\
+ & \left(\alpha-\alpha^{2}\right)\left(1-\frac{\Delta_{n}}{\delta_{n}}\right)^{\alpha-2}\left(\frac{m}{\alpha}+\frac{\Delta_{n}}{\delta_{n}}\right)^{m} \\
& \left.-2 \alpha m\left(1-\frac{\Delta_{n}}{\delta_{n}}\right)^{\alpha-1}\left(\frac{m}{\alpha}+\frac{\Delta_{n}}{\delta_{n}}\right)^{m}\right] \\
& {\left[\Gamma_{t}\left(1-\frac{\left|\Delta_{t}\right|}{\delta_{t}}\right)^{\beta}\left(\frac{n}{\beta}+\frac{\left|\Delta_{t}\right|}{\delta_{t}}\right)^{n}+\left\langle\phi_{t}-\phi_{n}\right\rangle\right] }
\end{aligned}
$$

Note that for the normal cohesive traction, the onset of the softening condition occurs when the normal separation is greater than the normal critical separation, which corresponds to the softening region.

The normal tangent stiffness within the softening region for each model is plotted in Figure 7 with the fracture parameters used in Section 3.1. For the Abaqus model (Figure 7(a)), positive stiffness is observed in a portion of the softening region, which results in the increase of the cohesive traction with respect to the increase of the cohesive separation. Because of such positive stiffness, the oscillation of the cohesive tractions shown in Figures 4 and 5 is expected. However, for the PPR model, Figure 7(b) clearly demonstrates that the stiffness within the softening region is always negative. This explains why the PPR 
cohesive tractions monotonically decrease within the softening region, as observed in Figures 4 and 5.

The tangent stiffness along the critical separation is indicated by a thick solid line (see Figure 7). For the model in Abaqus, before reaching the onset of crack initiation, the tangent stiffness is initially constant, i.e. the penalty stiffness (see Eq. (2)). When the effective separation reaches the critical separation, the magnitude and sign of the tangent stiffness change according to the ratio of the normal separation to the tangential separation. Thus, a discontinuous tangent stiffness within the cohesive interaction region is expected. However, the PPR model provides zero stiffness along the critical separation, which results in the continuous stiffness distribution within the cohesive interaction region.

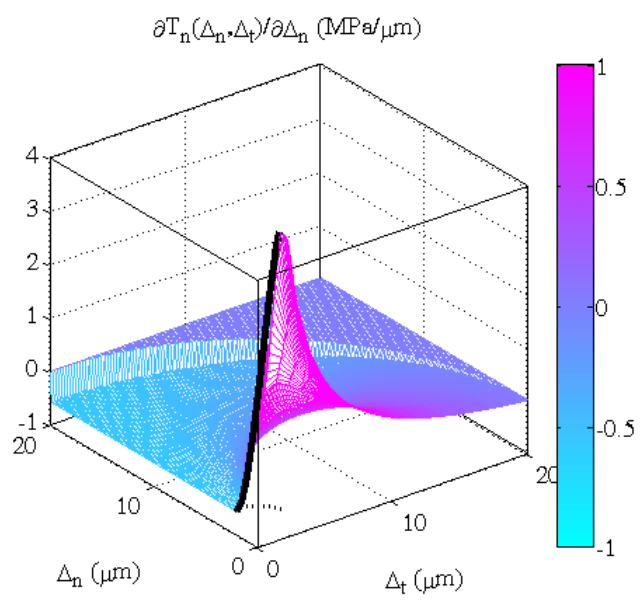

(a)

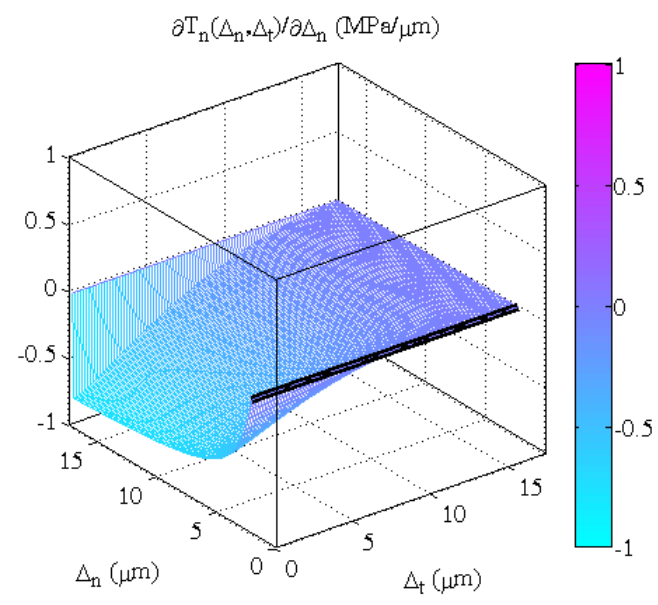

(b)

Fig. 7. Normal material tangent stiffness of (a) Abaqus model and (b) PPR model.

\subsection{Onset of softening for an arbitrary separation path}

In order to check the consistency of the Abaqus, PPR, and non-potential based models, the onset of softening is evaluated for an arbitrary separation path. The normal separation initially increases up to $0.5 \mu \mathrm{m}$ (denoted as Point $\mathbf{S}_{\mathbf{A}}$ ), and then the tangential separation increase up to $1 \mu \mathrm{m}$ (denoted as Point $\mathbf{S}_{\mathbf{B}}$ ). Afterwards, the normal separation increases up to the complete failure condition. For the given separation path, the traction-separation relationships of the Abaqus, PPR, and the non-potential based models result in similar cohesive traction variations but different positions of the onset of softening.

As shown in Figure 8, for the Abaqus, PPR, and non-potential based models, the normal traction initially increases within the elastic region while the normal separation increases. As the tangential separation increases from Point $\mathbf{S}_{\mathbf{A}}$ to Point $\mathbf{S}_{\mathbf{B}}$, the normal traction decreases, because the increase of tangential separation results in the decrease of the cohesive strength under mixed-mode condition. Then, while the normal separation increases, the cohesive traction reaches a maximum value and monotonically decreases to zero. The corresponding cohesive tractions of Abaqus, PPR and NP3 models are plotted using a black solid line, while the cohesive traction along the critical separation is indicated as a red dashed line within the normal traction-separation relationships, as shown in Figure 8. Note that the NP3 model is selected as a representative example of the non-potential based models by McGarry et al (2016). The cohesive traction along the critical separation is not plotted for the NP3 model (see Figure 8(c)) because the onset of softening is not clearly defined in this non-potential based model.

In the Abaqus model, the onset of softening is reached when the tangential separation increases from Point $\mathbf{S}_{\mathbf{A}}$ to Point $\mathbf{S}_{\mathbf{B}}$, which corresponds to the intersection of the red dashed line and the black solid line in Figure 8(a). After the onset of softening is reached, the cohesive normal traction predicted by the Abaqus model increases as the normal separation increases, as discussed previously.

In contrast to the Abaqus model, the onset of softening of the PPR model corresponds to the maximum traction, as shown by the intersection between the red dashed line and the black solid line in Figure 8(b). This leads to a decrease of the cohesive traction as the normal separation increases in the softening region, thus demonstrating consistency of the PPR model. 


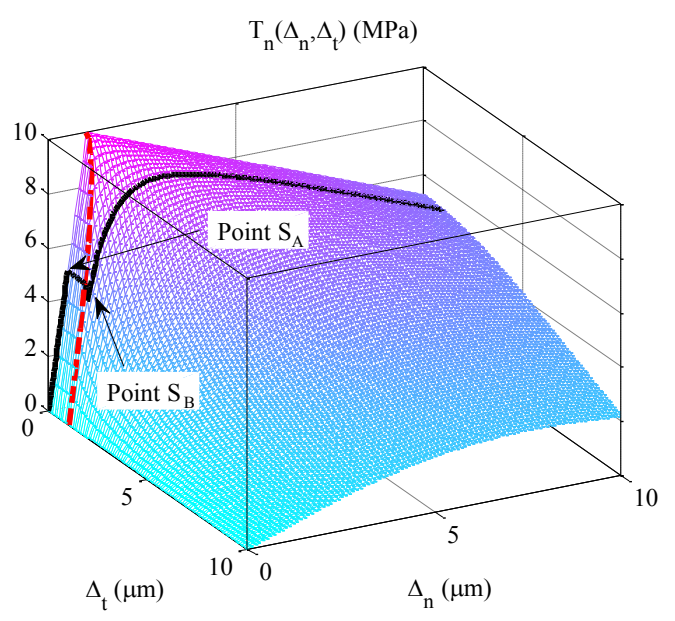

(a)

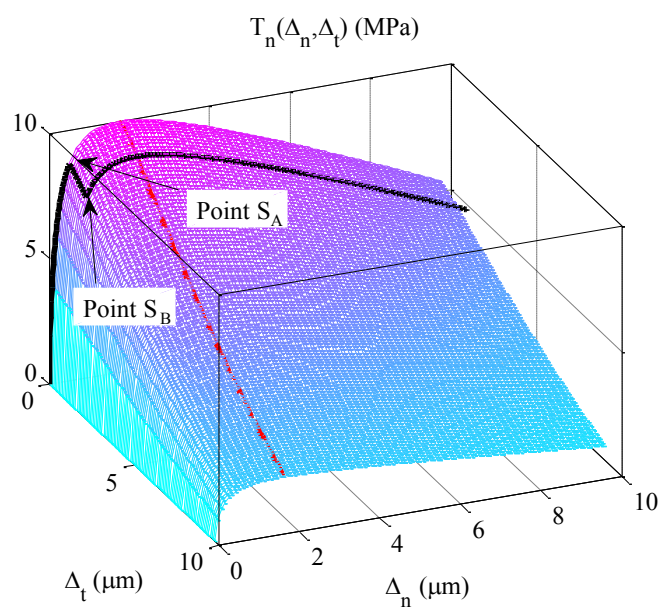

(b)

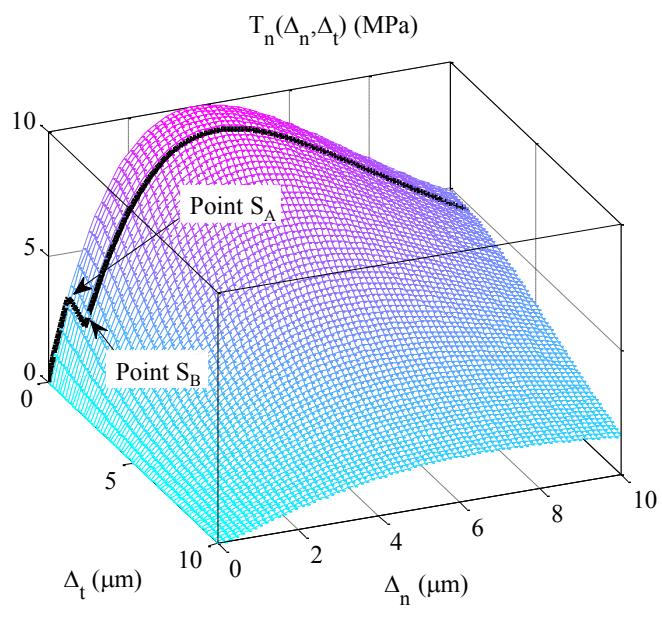

(c)

Fig. 8. Cohesive traction for a given separation path within the normal traction-separation relationships (a) Abaqus model, (b) PPR model, and (c) NP3 model.
For the non-potential NP3 model, the onset of softening is not clearly defined in the tractionseparation relationship, and thus it may be assumed on the basis of: (1) the sign of the rate of cohesive traction, or (2) the maximum cohesive traction. First, the onset of softening can be assumed when the sign of the rate of cohesive traction changes from positive to negative, which corresponds to Point $\mathbf{S}_{\mathbf{A}}$ in this example. After reaching Point $\mathbf{S}_{\mathbf{B}}$, the cohesive traction of the NP3 model increases up to $9.65 \mathrm{MPa}$ while the cohesive traction at Point $\mathbf{S}_{\mathbf{A}}$ is $3.23 \mathrm{MPa}$. Therefore, this assumption results in the onset of softening at a very low cohesive traction, which is not physical.

Alternatively, the onset of softening can be defined as a maximum cohesive traction in a separation history. However, in this case, one cannot identify the onset of softening before knowing the entire separation path, which is not possible in general computational simulations.

\section{Conclusions}

A comparative study of traction-separation relationships in cohesive zone models has been presented. Specifically, the traction-separation relationship in Abaqus is assessed in order to check the consistency of the constitutive model, and is compared with the traction-separation relationship of the PPR model. The model in Abaqus can provide non-physical behavior for certain separation paths because the model does not always provide a negative tangent stiffness within the softening region. Similar issues were observed with the so-called NP1, NP2 and NP3 models by McGarry et al. (2014). Thus, the cohesive traction can increase and decrease repeatedly while the separation monotonically increases under softening conditions. Although the Abaqus model may provide satisfactory results for certain classes of problems, the oscillation and the positive stiffness in the tractionseparation relation can adversely impact instances such as numerical stability, multiple crack interactions, and dissipated energy. In comparison, the PPR model does not display such non-physical behavior and guarantees the negative tangent stiffness within the softening region. In conclusion, traction-separation relationships should be carefully developed and selected for mixedmode failure investigations.

\section{Acknowledgements}

K. Park acknowledges support from the National Research Foundation (NRF) of Korea through grant number NRF-2015R1C1A1A02037663. G.H. Paulino acknowledges support from the US National Science 
Foundation (NSF) under grant number CMMI 1321661. The information presented in this paper is the sole opinion of the authors and does not necessarily reflect the views of the sponsoring agencies.

\section{References}

Abaqus (2013). Abaqus 6.13 Analysis User's Manual, SIMULIA, Providence, IR.

Benzeggagh, M. L., \& Kenane, M., (1996). Measurement of mixed-mode delamination fracture toughness of unidirectional glass/epoxy composites with mixed-mode bending apparatus. Composites Science and Technology 56 (4), 439-449.

Camanho, P. P., Davila, C. G., \& Moura, M. F. De. (2003). Numerical simulation of mixed-mode progressive delamination in composite materials. Journal of Composite Materials 37(16), 1415-1438.

Campilho, R. D. S. G., Banea, M. D., Neto, J. A. B. P., \& Da Silva, L. F. M. (2013). Modelling adhesive joints with cohesive zone models: Effect of the cohesive law shape of the adhesive layer. International Journal of Adhesion and Adhesives 44, 48-56.

Cerrone, A., Wawrzynek, P., Nonn, A., Paulino, G. H., \& Ingraffea, A. (2014). Implementation and verification of the Park-Paulino-Roesler cohesive zone model in 3D. Engineering Fracture Mechanics 120, 26-42.

Chandra, N., Li, H., Shet, C., \& Ghonem, H. (2002). Some issues in the application of cohesive zone models for metal-ceramic interfaces. International Journal of Solids and Structures 39(10), 2827-2855.

Elices, M., Guinea, G. V., Gomez, J., \& Planas, J. (2002). The cohesive zone model: advantages, limitations and challenges. Engineering Fracture Mechanics 69(2), 137-163.

Harper, P. W., \& Hallett, S. R. (2008). Cohesive zone length in numerical simulations of composite delamination. Engineering Fracture Mechanics 75(16), 4774-4792.

Jiang, W.-G., Hallett, S. R., Green, B. G., \& Wisnom, M. R. (2007). A concise interface constitutive law for analysis of delamination and splitting in composite materials and its application to scaled notched tensile specimens. International Journal for Numerical Methods in Engineering 69, 1982-1995.

Lee, M. J., Cho, T. M., Kim, W. S., Lee, B. C., \& Lee, J. J. (2010). Determination of cohesive parameters for a mixed-mode cohesive zone model. International Journal of Adhesion and Adhesives 30(5), 322-328.

Leon, S. E., Spring, D. W., \& Paulino, G. H. (2014). Reduction in mesh bias for dynamic fracture using adaptive splitting of polygonal finite elements. International Journal for Numerical Methods in Engineering 100, 555-576.

McGarry, J. P., Ó Máirtín, É., Parry, G., \& Beltz, G. E. (2014). Potential-based and non-potential-based cohesive zone formulations under mixed-mode separation and over-closure. Part I: Theoretical analysis. Journal of the Mechanics and Physics of Solids 63(1), 336-362.

Ó Máirtín, É., Parry, G., Beltz, G. E., \& McGarry, J. P. (2014). Potential-based and non-potential-based cohesive zone formulations under mixed-mode separation and over-closure. Part II: Finite element applications. Journal of the Mechanics and Physics of Solids 63(1), 363-385.

De Morais, A. B., \& de Moura, M. F. S. F. (2006). Evaluation of initiation criteria used in interlaminar fracture tests. Engineering Fracture Mechanics 73(16), 2264-2276.

Needleman, A. (1987). A continuum model for void nucleation by inclusion debonding. Journal of Applied Mechanics - Transactions of the ASME 54 (3), 525-531.

Needleman, A. (1990). An analysis of tensile decohesion along an interface. Journal of the Mechanics and Physics of Solids 38 (3), 289-324.

Neto, P., Alfaiate, J., \& Vinagre, J. (2016). Assessment of the dependence of CFRP-concrete behaviour on the width of the bonded materials. Composites Part B: Engineering 91, 448-457.

Park, K., Paulino, G. H., \& Roesler, J. R. (2009). A unified potential-based cohesive model of mixed-mode fracture. Journal of the Mechanics and Physics of Solids 57(6), 891-908.

Park, K., Paulino, G. H., \& Roesler, J. (2010). Cohesive fracture model for functionally graded fiber reinforced concrete. Cement and Concrete Research 40(6), 956-965.

Park, K., \& Paulino, G. H. (2012). Computational implementation of the PPR potential-based cohesive model in ABAQUS: Educational perspective. Engineering Fracture Mechanics 93, 239-262.

Park, K., \& Paulino, G. H. (2013). Cohesive zone models: a critical review of traction-separation relationships across fracture surfaces. Applied Mechanics Reviews 64(6), 060802.

Paulino, G. H., Park, K., Celes, W., \& Espinha, R. (2010). Adaptive dynamic cohesive fracture simulation using nodal perturbation and edge-swap operators. International Journal for Numerical Methods in Engineering 84, 1303-1343.

Sørensen, B. F., Goutianos, S., \& Jacobsen, T. K. (2009). Strength scaling of adhesive joints in polymer- 
matrix composites. International Journal of Solids and Structures 46(3-4), 741-761.

Spring, D. W., Leon, S. E., \& Paulino, G. H. (2014). Unstructured polygonal meshes with adaptive refinement for the numerical simulation of dynamic cohesive fracture. International Journal of Fracture 189(1), 33-57.

Spring, D. W., \& Paulino, G. H. (2014). A growing library of three-dimensional cohesive elements for use in ABAQUS. Engineering Fracture Mechanics 126, 190-216.

Turon, A., Camanho, P. P., Costa, J., \& Dávila, C. G. (2006). A damage model for the simulation of delamination in advanced composites under variablemode loading. Mechanics of Materials 38(11), 10721089.

Tvergaard, V. (1990). Effect of fiber debonding in a whisker-reinforced metal. Materials Science and Engineering, A125, 203-213.

Tvergaard, V., \& Hutchinson, J. W. (1992). The relation between crack growth resistance and fracture process parameters in elastic-plastic solids. Journal of the Mechanics and Physics of Solids 40(6), 1377-1397.

Wittmann, F. H., Rokugo, K., Brühwiler, E., Mihashi, H., \& Simonin, P. (1988). Fracture energy and strain softening of concrete as determined by means of compact tension specimens. Materials and Structures 21(1), 21-32.

Xu, X. P., \& Needleman, A., (1993). Void nucleation by inclusion debonding in a crystal matrix. Modelling and Simulation in Materials Science and Engineering 1 (2), 111-132.

Yang, Q. D., \& Thouless, M. D. (2001). Mixed-mode fracture analyses of plastically-deforming adhesive. International Journal of Fracture 110, 175-187.

Yang, Z. J., Su, X. T., Chen, J. F., \& Liu, G. H. (2009). Monte Carlo simulation of complex cohesive fracture in random heterogeneous quasi-brittle materials. International Journal of Solids and Structures 46(17), 3222-3234.

\section{Appendix A. Nomenclature}

The following symbols are used in this paper.

$$
\begin{array}{ll}
T_{n}, T_{t} & \text { normal and tangential tractions } \\
K_{p} & \text { penalty stiffness } \\
d & \text { scalar damage variable } \\
m, n & \text { characteristic parameters in the PPR model } \\
\Delta_{n}, \Delta_{t} & \text { normal and tangential separations } \\
\bar{\Delta} & \text { effective separation }
\end{array}
$$

$\Gamma_{n}, \Gamma_{t} \quad$ characteristic parameters in the PPR model

$\Psi \quad$ potential for cohesive fracture

$\alpha, \beta \quad$ shape parameters in the PPR model

$\alpha_{m}, \beta_{m}$ parameters for weight of the mode-mixity

$\alpha_{r} \quad$ parameter for the rate of damage evolution

$\beta_{e} \quad$ ratio of tangential separation to normal separation

$\eta \quad$ parameter for variation of fracture toughness

$\overline{\delta_{c}} \quad$ effective critical separation

$\overline{\delta_{f}} \quad$ effective complete failure separation

$\delta_{n}, \delta_{t} \quad$ characteristic length scale parameters

$\delta_{n c}, \delta_{t c}$ characteristic lengths for critical separations

$\lambda_{n}, \lambda_{t} \quad$ initial slope indicators

$\sigma_{\max }, \tau_{\max }$ normal and tangential cohesive strengths

$\phi_{n}, \phi_{t}$ normal and tangential fracture energies 